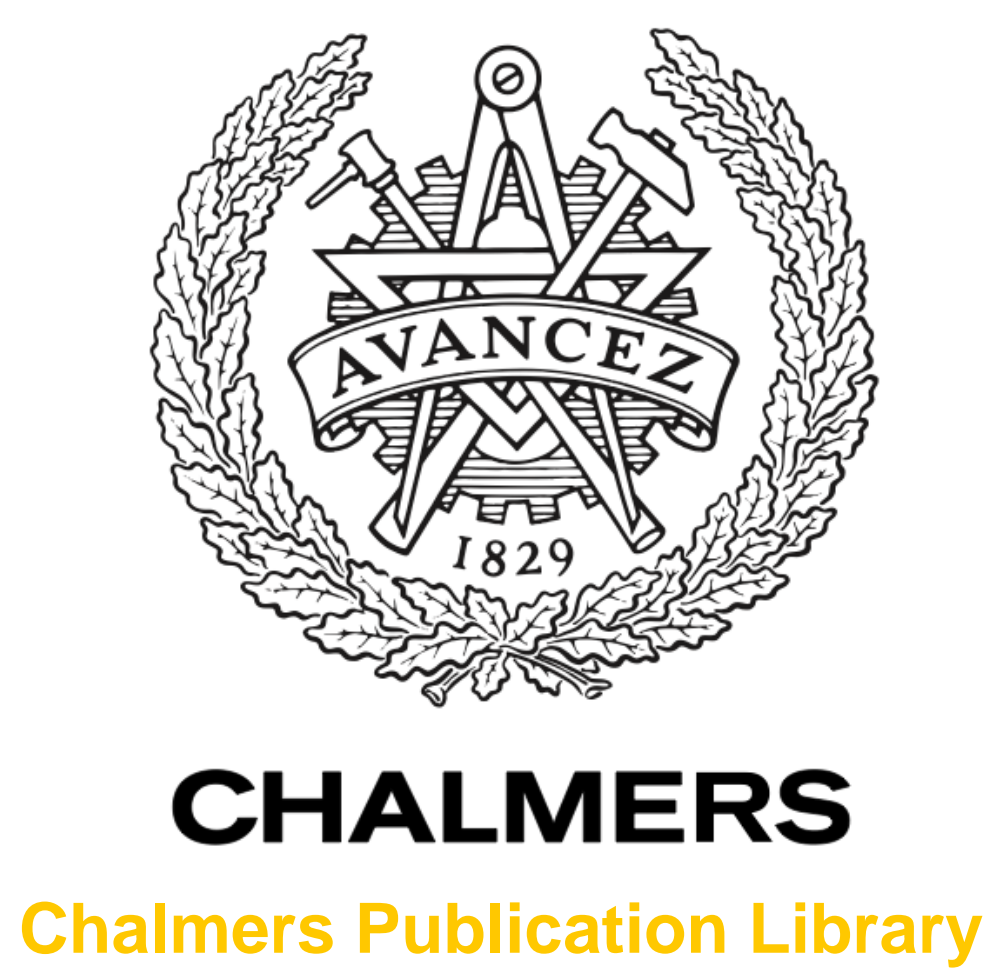

Spectrum sharing via HARQ feedback and adaptive power allocation

This document has been downloaded from Chalmers Publication Library (CPL). It is the author's version of a work that was accepted for publication in:

2014 IEEE Wireless Communications and Networking Conference, WCNC 2014; Istanbul; Turkey; 6 April 2014 through 9 April 2014 (ISSN: 1525-3511)

Citation for the published paper:

Makki, B. ; Eriksson, T. ; Svensson, T. (2014) "Spectrum sharing via HARQ feedback and adaptive power allocation". 2014 IEEE Wireless Communications and Networking Conference, WCNC 2014; Istanbul; Turkey; 6 April 2014 through 9 April 2014 pp. 10241029.

http://dx.doi.org/10.1109/WCNC.2014.6952249

Downloaded from: http://publications.lib.chalmers.se/publication/192546

Notice: Changes introduced as a result of publishing processes such as copy-editing and formatting may not be reflected in this document. For a definitive version of this work, please refer to the published source. Please note that access to the published version might require a subscription.

Chalmers Publication Library (CPL) offers the possibility of retrieving research publications produced at Chalmers University of Technology. It covers all types of publications: articles, dissertations, licentiate theses, masters theses, conference papers, reports etc. Since 2006 it is the official tool for Chalmers official publication statistics. To ensure that Chalmers research results are disseminated as widely as possible, an Open Access Policy has been adopted.

The CPL service is administrated and maintained by Chalmers Library. 


\title{
Spectrum sharing via HARQ feedback and adaptive power allocation
}

\author{
Behrooz Makki, Thomas Eriksson and Tommy Svensson \\ Department of Signals and Systems, Chalmers University of Technology, Gothenburg, Sweden \\ Email: \{behrooz.makki, thomase, tommy.svensson\}@chalmers.se
}

\begin{abstract}
Recently, substantial attention has been paid to improve the spectral efficiency of communication setups using different spectrum sharing techniques. This paper studies the throughput of spectrum sharing channels utilizing hybrid automatic repeat request (HARQ) protocols. Considering different HARQ schemes, the unlicensed user throughput is obtained under an outage probability constraint for the licensed user. The outage-limited throughput is obtained for both independent and spatially-correlated fading conditions, where there is spatial dependency between the fading coefficients. The results show that, using HARQ and adaptive power allocation, the maximum throughput is achieved by combination of simultaneous transmission and interference-avoiding spectrum sharing paradigms. The performance of the spectrum sharing networks is not sensitive to spatial correlation, within the practical range, and the throughput changes are negligible at low/moderate correlations. Finally, there is considerable potential for data transmission of the unlicensed user with limited performance degradation of the licensed user.
\end{abstract}

\section{INTRODUCTION}

Spectrum is a scarce valuable resource in today's wireless communication networks; with ever-increasing number of wireless devices communicating at high data rates, there is growing demand for spectrum resources. This point has led to complaints about spectrum shortage which is expected to grow even more in the coming years.

To tackle the spectrum shortage problem, several dynamic spectrum management solutions have been proposed among which spectrum sharing is one of the most promising ones [1][7]. In a spectrum sharing network, unlicensed secondary users (SUs) are permitted to work within the spectrum resources of licensed primary users (PUs) as long as the PUs quality-ofservice $(\mathrm{Q} O S)$ requirements are satisfied. In general, there are two methods for spectrum sharing. In a method widely referred to as the interference-avoiding paradigm [3]-[5], the SUs are not permitted to work within the PUs activation period. In another scheme, normally denoted simultaneous or controlled transmission [6]-[17], a SU can simultaneously coexist with a PU as long as it works under a certain interference level imposed by the PU QoS requirements.

From another perspective, hybrid automatic repeat request (HARQ) is a well-known approach applied in wireless networks to increase the data transmission reliability and efficiency [12]-[20]. The main idea behind the HARQ techniques is to reduce the data outage probability or increase the throughput by retransmitting the data that underwent bad channel conditions. Therefore, it is interesting to study the performance of the spectrum sharing networks in the presence of HARQ.
The effect of optimal power allocation on the performance of the HARQ-based single-user networks has been studied in, e.g., [18]-[20]. Utilizing HARQ in spectrum sharing networks has recently attracted considerable attention, e.g., [12]-[17]. In [12], [13], the SU works as a relay helping the PU, which uses incremental redundancy (INR) HARQ. Also, in [14], [15] the INR HARQ is exploited by the PU for increasing its protection against the SU interferences. Finally, [16], [17] consider different HARQ protocols for the SU, to maximize the SU average rate under different $\mathrm{PU}$ peak/average interference constraints.

In this paper, we study the data transmission efficiency of an HARQ-based spectrum sharing network. The problem is cast in form of maximizing the SU long-term throughput with an outage probability constraint for the PU. The results are obtained for both independent and spatially-correlated fading conditions, where there is spatial dependency between the fading coefficients. Also, we provide connection between the simultaneous transmission and interference-avoiding paradigms by demonstrating the cases where each of these methods are optimal, in terms of throughput. Different HARQ protocols are exploited by the PU. The SU transmission rates and powers are optimized, in terms of throughput, such that the PU outage probability constraint is satisfied.

The problem setup of the paper is different from the ones in [12]-[15] because, as opposed to [12]-[15], the SU does not work as a relay and also we consider adaptive power allocation and different QoS requirements. Moreover, in contrast to [16], $[17], 1)$ we consider the HARQ feedback for the PU, 2) the results are obtained for different SU and PU QoS requirements and 3) there is no (im)perfect channel state information (CSI) available at the transmitters. The differences in the system model and the QoS requirements make the problem solved in this paper completely different from the ones addressed in [12]-[20], leading to different analytical and numerical results, as well as to different conclusions.

The theoretical and numerical results show that with HARQ and adaptive power allocation, the maximum outage-limited throughput is achieved by combination of the simultaneous transmission and interference-avoiding paradigms. Also, considering the practical range of spatial correlations, the data transmission efficiency of the spectrum sharing networks is not sensitive to spatial correlation. Moreover, we present analytical approximations for the performance analysis of the HARQbased spectrum sharing networks. Finally, as demonstrated in the simulations, implementation of HARQ protocols and 
adaptive power allocation leads to considerable performance improvement in spectrum sharing networks.

\section{SYSTEM MODEL}

As illustrated in Fig.1, we consider a standard spectrum sharing network where two primary and secondary users share the same narrow-band frequency with bandwidth $W$. With no loss of generality, we set $W=1$. Let $H_{m}^{\mathrm{pp}}, H_{m}^{\mathrm{ps}}, H_{m}^{\mathrm{sp}}$ and $H_{m}^{s s}$ be the fading variables of the PU-PU, PU-SU, SUPU and SU-SU links at the $m$-th time slot, respectively. Correspondingly, we define $G_{m}^{\mathrm{pp}} \doteq\left|H_{m}^{\mathrm{pp}}\right|^{2}, G_{m}^{\mathrm{ps}} \doteq\left|H_{m}^{\mathrm{ps}}\right|^{2}$, $G_{m}^{\mathrm{sp}} \doteq\left|H_{m}^{\mathrm{sp}}\right|^{2}$ and $G_{m}^{\mathrm{ss}} \doteq\left|H_{m}^{\mathrm{ss}}\right|^{2}$ which are denoted channel gains in the following. Also, the gains probability density functions (pdf:s) are represented by $f_{G^{\mathrm{pp}}}, f_{G^{\mathrm{ps}}}, f_{G^{\mathrm{sp}}}$ and $f_{G^{\mathrm{ss}}}$, respectively. In Section III, we focus on independent Rayleighfading channels where the fading coefficients follow, e.g., $H^{\mathrm{pp}} \sim \mathcal{C} \mathcal{N}\left(0, \frac{1}{\lambda^{\mathrm{pp}}}\right)$. Thus, the gains pdf are given by, e.g., $f_{G \mathrm{pp}}(g)=\lambda^{\mathrm{pp}} e^{-\lambda^{\mathrm{pp}} g}, g \geq 0$. The results will be extended to the spatially-correlated fading channels in Section IV. The complex white Gaussian noises $Z_{m}^{\mathrm{p}}$ and $Z_{m}^{\mathrm{s}}$ added at the PU and SU receivers, are supposed to have distributions $\mathcal{C N}(0,1)$. In this way, the channel outputs at the $m$-th time slot can be stated as

$$
\left\{\begin{array}{c}
Y_{m}^{\mathrm{p}}=X_{m}^{\mathrm{p}} H_{m}^{\mathrm{pp}}+X_{m}^{\mathrm{s}} H_{m}^{\mathrm{sp}}+Z_{m}^{\mathrm{p}} \\
Y_{m}^{\mathrm{s}}=X_{m}^{\mathrm{s}} H_{m}^{\mathrm{ss}}+X_{m}^{\mathrm{p}} H_{m}^{\mathrm{ps}}+Z_{m}^{\mathrm{s}},
\end{array}\right.
$$

where $X_{m}^{\mathrm{p}}$ and $X_{m}^{\mathrm{s}}$ are the PU and SU input messages, respectively, and $Y_{m}^{\mathrm{p}}$ and $Y_{m}^{\mathrm{s}}$ denote their corresponding outputs. Imposed by the power amplifier and hardware constraints, we consider a peak power constraint for the users, e.g., $T_{m}^{\mathrm{s}} \leq T^{\text {peak }}, \forall m$, where $T_{m}^{\mathrm{s}}$ is the $\mathrm{SU}$ transmission power at the $m$-th slot and $T^{\text {peak }}$ denotes the peak power constraint.

We focus on block-fading channels where the channels remain fixed during the channel coherence time and then change independently according to their corresponding distributions. In each block, the channel gains are supposed to be known by the receivers which is an acceptable assumption in blockfading channels [12]-[20]. The PU utilizes different HARQ protocols with a maximum of $M$ retransmission rounds, i.e., the PU codewords are (re)transmitted a maximum of $M+1$ times. Particularly, two different HARQ protocols are considered for the PU:

1) Repetition time diversity (RTD). This scheme belongs to the diversity combining category of HARQ protocols [19], [20] where the same data is repeated in the (re)transmission rounds and, in each round, the receiver performs maximum ratio combining (MRC) of all received signals.

2) Incremental Redundancy (INR). The INR belongs to the category of code combining protocols [20]. Here, a codeword is sent with an aggressive rate in the first round. Then, if the receiver cannot decode the initial codeword, further parity bits are sent in the next retransmission rounds and in each round the receiver decodes the data based on all received signals.

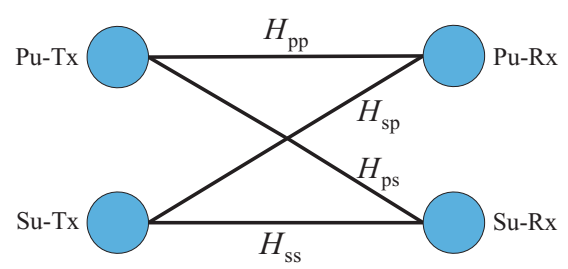

Figure 1. Channel model.

No HARQ feedback is considered for the SU. However, the SU listens to the PU acknowledgement/non-acknowledgement (ACK/NACK) signals, and adapts its data transmission rate and power based on the PU HARQ feedback bits. In this way, with a peak power constraint for the SU, the SU transmission powers/rates are optimized, depending on the PU message decoding status, such that the SU throughput is maximized for a given PU outage probability constraint.

\section{THEORETICAL RESULTS}

Let the PU transmission power in the $m$-th retransmission round of the HARQ protocol be $T_{m}^{\mathrm{p}}$. Also, define $R^{\mathrm{p}}$ as the PU initial data transmission rate in an HARQ-based packet, where a packet is defined as the transmission of a codeword along with all of its possible retransmission rounds. In the following, we first investigate the system performance for the RTD HARQ protocol and then the results are extended to the case with the INR HARQ. Here, the results are obtained for independent Rayleigh-fading channels. We study the effects of spatial correlation in Section IV.

\section{A. RTD HARQ protocol}

Utilizing the RTD HARQ, the same codeword is (re)transmitted by the PU in each (re)transmission round and the PU receiver performs MRC of the received signals. Therefore, after $m$ data (re)transmission rounds the PU equivalent data rate reduces to $\frac{R^{\mathrm{p}}}{m}$ and the PU received signal-tointerference-and-noise ratio (SINR) increases to

$$
\phi_{(m)}^{\mathrm{p}}=\sum_{i=1}^{m} \operatorname{SINR}_{i}^{\mathrm{p}}=\sum_{i=1}^{m} \frac{T_{i}^{\mathrm{p}} G_{i}^{\mathrm{pp}}}{1+T_{i}^{\mathrm{s}} G_{i}^{\mathrm{sp}}} .
$$

Here, $T_{i}^{\mathrm{s}}$ denotes the SU transmission power in the PU's $i$-th retransmission round.

The data is correctly decoded by the PU receiver at the end of the $m$-th round (and not before) if 1) the receiver has not decoded the data before, i.e., $\log \left(1+\sum_{i=1}^{n} \frac{T_{i}^{\mathrm{p}} G_{i}^{\mathrm{pp}}}{1+T_{i}^{\mathrm{sp}} G_{i}^{\mathrm{sp}}}\right)<$ $R^{\mathrm{p}}, \forall n<m$, and 2) (re)transmitting the data in the $m$ th slot, the $\mathrm{PU}$ receiver can decode the codeword, i.e., $\log \left(1+\sum_{i=1}^{m} \frac{T_{i}^{\mathrm{p}} G_{i}^{\mathrm{pp}}}{1+T_{i}^{\mathrm{s}} G_{i}^{\mathrm{sp}}}\right) \geq R^{\mathrm{p}}$. Hence, the probability that the PU message is decoded at $m$-th round (and not before) is

$$
\begin{array}{r}
\operatorname{Pr}\left(A_{m}\right)^{\mathrm{p}, \mathrm{RTD}}=\operatorname{Pr}\left(\log \left(1+\sum_{i=1}^{m-1} \frac{T_{i}^{\mathrm{p}} G_{i}^{\mathrm{pp}}}{1+T_{i}^{\mathrm{s}} G_{i}^{\mathrm{sp}}}\right)<R^{\mathrm{p}} \leq\right. \\
\left.\log \left(1+\sum_{i=1}^{m} \frac{T_{i}^{\mathrm{p}} G_{i}^{\mathrm{pp}}}{1+T_{i}^{\mathrm{s}} G_{i}^{\mathrm{sp}}}\right)\right) .
\end{array}
$$

Note that in (3) we have used the fact that with an equivalent SINR $x$ the maximum decodable transmission rate is 
$\frac{1}{m} \log (1+x)$ if a codeword is repeated $m$ times. Also, the PU outage probability is obtained by

$$
\begin{aligned}
& \operatorname{Pr}(\text { Outage })^{\mathrm{p}, \mathrm{RTD}}=1-\sum_{i=1}^{M+1} \operatorname{Pr}\left(A_{m}\right)^{\mathrm{p}, \mathrm{RTD}} \\
& =\operatorname{Pr}\left(\log \left(1+\sum_{i=1}^{M+1} \frac{T_{i}^{\mathrm{p}} G_{i}^{\mathrm{pp}}}{1+T_{i}^{\mathrm{s}} G_{i}^{\mathrm{sp}}}\right)<R^{\mathrm{p}}\right) .
\end{aligned}
$$

Finally, the probability that the PU data retransmission continues until the end of the $m$-th round is given by

$\operatorname{Pr}\left(B_{m}\right)^{\mathrm{p}, \mathrm{RTD}}= \begin{cases}\operatorname{Pr}\left(A_{m}\right)^{\mathrm{p}, \mathrm{RTD}} & \text { if } m=1, \ldots, M, \\ 1-\sum_{m=1}^{M} \operatorname{Pr}\left(A_{m}\right)^{\mathrm{p}, \mathrm{RTD}} & \text { if } m=M+1,\end{cases}$

where $\sum_{m=1}^{M+1} \operatorname{Pr}\left(B_{m}\right)^{\mathrm{p}, \mathrm{RTD}}=1$, as a maximum of $M+1$ (re)transmission rounds is considered for the PU.

Given that the PU is in the $m$-th (re)transmission round, the SU transmission rate and power are set to $R_{m}^{\mathrm{s}}$ and $T_{m}^{\mathrm{s}}$, respectively. The SU codeword sent at the PU $m$-th (re)transmission round is correctly decoded by the SU receiver if $\log \left(1+\frac{T_{m}^{\mathrm{s}} G_{m}^{\mathrm{ss}}}{1+T_{m}^{\mathrm{p}} G_{\mathrm{m}}^{\mathrm{ps}}}\right) \geq R_{m}^{\mathrm{s}}$. This is based on the fact that no HARQ is used by the SU and the SU received SINR at the $m$-th retransmission round of the PU is $\operatorname{SINR}_{m}^{\mathrm{s}}=\frac{T_{m}^{\mathrm{s}} G_{m}^{\mathrm{ss}}}{1+T_{m}^{\mathrm{p}} G_{m}^{\mathrm{ps}}}$.

In this way, the SU long-term throughput is found as

$$
\begin{aligned}
& \eta^{\mathrm{s}, \mathrm{RTD}}= \\
& \sum_{m=1}^{M+1} \operatorname{Pr}\left(B_{m}\right)^{\mathrm{p}, \mathrm{RTD}}\left(\sum_{n=1}^{m} R_{n}^{\mathrm{s}} \operatorname{Pr}\left(R_{n}^{\mathrm{s}} \leq \log \left(1+\frac{T_{n}^{\mathrm{s}} G_{n}^{\mathrm{ss}}}{1+T_{n}^{\mathrm{p}} G_{n}^{\mathrm{ps}}}\right)\right)\right. \\
& =\sum_{n=1}^{M+1} R_{n}^{\mathrm{s}}\left(\frac{e^{-\frac{\lambda^{\mathrm{ss}} \alpha_{n}^{\mathrm{s}}}{T_{n}^{\mathrm{s}}}}}{1+\frac{\lambda^{\mathrm{ss}} T_{n}^{\mathrm{p}}}{\lambda^{\mathrm{ps}} T_{n}^{\mathrm{s}}} \alpha_{n}^{\mathrm{s}}}\right)\left(1-\sum_{i=1}^{n-1} \operatorname{Pr}\left(B_{i}\right)^{\mathrm{p}, \mathrm{RTD}}\right) \\
& =\sum_{n=1}^{M+1} R_{n}^{\mathrm{s}}\left(\frac{e^{-\frac{\lambda^{\mathrm{ss}} \alpha_{n}^{\mathrm{s}}}{T_{n}^{\mathrm{s}}}}}{1+\frac{\lambda^{\mathrm{ss}} T_{n}^{\mathrm{p}}}{\lambda^{\mathrm{ps}} T_{n}^{\mathrm{s}}} \alpha_{n}^{\mathrm{s}}}\right) \Gamma_{n-1}^{\mathrm{p}, \mathrm{RTD}}, \Gamma_{0}^{\mathrm{p}, \mathrm{RTD}}=1, \\
& \Gamma_{n}^{\mathrm{p}, \mathrm{RTD}}=\operatorname{Pr}\left(\log \left(1+\sum_{i=1}^{\mathrm{p}} \frac{T_{i}^{\mathrm{p}} G_{i}^{\mathrm{pp}}}{1+T_{i}^{\mathrm{s}} G_{i}^{\mathrm{sp}}}\right)<R^{\mathrm{p}}\right), \alpha_{m}^{\mathrm{s}}=e^{R_{m}^{\mathrm{s}}}-1
\end{aligned}
$$

which is the expectation of the SU achievable rates for different PU data (re)transmission conditions. Here, (6) comes from some manipulations and the fact that the random variable $\Theta_{m}^{\mathrm{s}}=\frac{T_{m}^{\mathrm{s}} G_{m}^{\mathrm{ss}}}{1+T_{m}^{\mathrm{p}} G_{m}^{\mathrm{ps}}}$ follows the cdf

$$
\begin{aligned}
& F_{\Theta_{m}^{\mathrm{s}}}(\theta)=\operatorname{Pr}\left(\frac{T_{m}^{\mathrm{s}} G_{m}^{\mathrm{ss}}}{1+T_{m}^{\mathrm{p}} G_{m}^{\mathrm{ps}}} \leq \theta\right) \\
& =\int_{0}^{\infty} f_{G^{\mathrm{ps}}}(x) \operatorname{Pr}\left(G_{m}^{\mathrm{ss}} \leq \frac{\theta}{T_{m}^{\mathrm{s}}}\left(1+T_{m}^{\mathrm{p}} x\right)\right) \mathrm{d} x \\
& =\int_{0}^{\infty} \lambda^{\mathrm{ps}} e^{-\lambda^{\mathrm{ps}} x}\left(1-e^{-\frac{\lambda^{\mathrm{ss}} \theta}{T_{m}^{\mathrm{s}}}\left(1+T_{m}^{\mathrm{p}} x\right)}\right) \mathrm{d} x=1-\frac{e^{-\frac{\lambda^{\mathrm{ss}} \theta}{T_{m}^{\mathrm{s}}}}}{1+\frac{\lambda^{\mathrm{ss}} T_{m}^{\mathrm{p}}}{\lambda^{\mathrm{p}} T_{m}^{\mathrm{s}}}} .
\end{aligned}
$$

In this way, considering an outage probability constraint $\operatorname{Pr}(\text { Outage })^{\text {p,RTD }} \leq \pi$ for the PU and a peak power constraint
$T_{m}^{\mathrm{s}} \leq T^{\text {peak }}, \forall m$, for the SU, the SU throughput maximization problem is represented as

$$
\begin{aligned}
& \max _{\forall T_{m}^{\mathrm{s}}, R_{m}^{\mathrm{s}}} \sum_{n=1}^{M+1} R_{n}^{\mathrm{s}}\left(\frac{e^{-\frac{\lambda^{\mathrm{ss}} \alpha_{n}^{\mathrm{s}}}{T_{n}^{\mathrm{s}}}}}{1+\frac{\lambda^{\mathrm{ss}} T_{n}^{\mathrm{p}}}{\lambda^{\mathrm{s}} T_{n}^{\mathrm{s}}} \alpha_{n}^{\mathrm{s}}}\right) \Gamma_{n-1}^{\mathrm{p}, \mathrm{RTD}} \\
& \text { s.t. } \operatorname{Pr}(\text { Outage })^{\mathrm{p}, \mathrm{RTD}} \leq \pi \quad \& \quad T_{m}^{\mathrm{s}} \leq T^{\text {peak }}, \forall m,
\end{aligned}
$$

which, depending on the number of retransmissions, can be solved numerically or analytically. The following theorem provides an optimal power allocation rule for the SU under the PU outage-limited condition. The theorem is of interest because it provides connection between the simultaneous transmission and interference-avoiding paradigms of spectrum sharing systems.

Theorem 1: For a given PU outage probability constraint, there is a threshold for the order of the PU retransmission rounds below which simultaneous transmission is the optimal transmission scheme, in terms of the SU throughput. However, for the PU retransmission round orders higher than the threshold, interference-avoiding is the optimal data transmission strategy, in terms of (8). That is, to maximize the SU outagelimited throughput, the SU should work in a time division multiple access (TDMA) fashion, determined by the PU QoS requirement.

Proof. For simplicity, consider a constant transmission power for the PU, i.e., $T_{m}^{\mathrm{p}}=T^{\mathrm{p}}, \forall m$ (as illustrated in the following, this is not a necessary assumption). In terms of the PU outage probability, (4) does not imply any preference between order of the SU transmission powers, i.e., two SU transmission powers $T_{m}^{\mathrm{s}}$ and $T_{n}^{\mathrm{s}}$ are interchangeable in (4). However, using (6), the contribution of the power term $T_{m-1}^{\mathrm{s}}$ on the SU throughput is higher than the one for $T_{m}^{\mathrm{s}}$. This is because $\Gamma_{m-1}^{p} \geq \Gamma_{m}^{\mathrm{p}}$, i.e., $\Gamma_{m}^{\mathrm{p}}$ is a decreasing function of $m$ (please see (6)). Thus, with the same transmission rates $R_{m-1}^{\mathrm{s}}=R_{m}^{\mathrm{s}}$, the SU throughput is increased if $T_{m-1}^{\mathrm{s}}>T_{m}^{\mathrm{s}}$. Hence, in the optimal case, there is a threshold $\mathcal{M}$ for the order of the PU retransmission rounds where the maximum SU throughput is achieved by $T_{m}^{\mathrm{s}}>0, m \leq \mathcal{M}$, and $T_{m}^{\mathrm{s}}=0, m>\mathcal{M}$. In fact, with the same arguments, it is found that in the optimal case we have $T_{m}^{\mathrm{s}}=T^{\text {peak }}, m<\mathcal{M}, T_{\mathcal{M}}^{\mathrm{s}} \in\left(0, T^{\text {peak }}\right]$ and $T_{m}^{\mathrm{s}}=0, m>\mathcal{M}$. In words, the maximum throughput is achieved as follows. The SU sends the data with the maximum power $T_{m}^{\mathrm{s}}=T^{\text {peak }}$ in the first PU retransmission rounds, i.e., in $m<\mathcal{M}$, (simultaneous transmission paradigm). Then, the transmission power is set to $T_{\mathcal{M}}^{\mathrm{s}} \in\left(0, T^{\text {peak }}\right]$ in the $\mathcal{M}$-th round (simultaneous transmission paradigm) and the SU is switched off in rounds $m>\mathcal{M}$ (interference-avoiding paradigm), so that the PU outage probability constraint is satisfied. The value of $\mathcal{M}$ depends on, e.g., PU (re)transmission powers, the SU peak power constraint and the PU outage probability constraint.

Considering single-user networks, it has been previously shown that, to minimize the outage probability of an HARQbased channel, the optimal (re)transmission powers should be an increasing function of $m$ [21]. That is, in contrast to the $\mathrm{SU}$, the optimal HARQ-based retransmission powers of the 
PU increase with $m$. In this way, with the same arguments as before and using $T_{m-1}^{\mathrm{p}} \leq T_{m}^{\mathrm{p}}, \forall m$, Theorem 1 can be reproved in the case with optimal PU power allocation.

Depending on the number of retransmissions, we may need to calculate the probabilities, e.g., $\Gamma_{m}^{\text {p,RTD }}$ numerically. The following lemma provides an approximation for the probability terms at low SU signal-to-noise ratios (SNRs).

Lemma 1: The low-SNR system performance can be approximated via the following approximation

$$
F_{\Theta_{m}^{\mathrm{p}}}(\omega) \rightarrow F_{\Omega_{m}^{p}}(\omega)=1-e^{-\beta_{m} \omega}, \beta_{m}=\frac{\lambda^{\mathrm{pp}}}{T_{m}^{\mathrm{p}}}+\frac{\lambda^{\mathrm{pp}} T_{m}^{\mathrm{s}}}{\lambda^{\mathrm{sp}} T_{m}^{\mathrm{p}}} .
$$

Proof. Using $\frac{1}{1+x} \rightarrow e^{-x}$ for small $x$ 's, the cdf $F_{\Theta_{m}^{\mathrm{p}}}(\omega)=$ $\operatorname{Pr}\left(\frac{T_{m}^{\mathrm{p}} G_{m}^{\mathrm{pp}}}{1+T_{m}^{\mathrm{s}} G_{m}^{\mathrm{sp}}} \leq \omega\right)=1-\frac{e^{-\frac{\lambda^{\mathrm{pp}} \omega}{T_{m}^{p}}}}{1+\frac{\lambda_{p}^{\mathrm{p}} T_{m}^{\mathrm{s}}}{\lambda^{\mathrm{sp}} T_{m}^{\mathrm{p}}} \omega}$ is approximated by

$$
F_{\Theta_{m}^{\mathrm{p}}}(\omega) \simeq F_{\Omega_{m}^{p}}(\omega)=1-e^{-\beta_{m} \omega}, \beta_{m}=\frac{\lambda^{\mathrm{pp}}}{T_{m}^{\mathrm{p}}}+\frac{\lambda^{\mathrm{pp}} T_{m}^{\mathrm{s}}}{\lambda^{\mathrm{sp}} T_{m}^{\mathrm{p}}} .
$$

Thus, the probabilities $\Gamma_{n}^{\mathrm{p}, \mathrm{RTD}}, \operatorname{Pr}(\text { Outage })^{\mathrm{p}, \mathrm{RTD}}=\Gamma_{M+1}^{\mathrm{p}, \mathrm{RTD}}$ and $\operatorname{Pr}\left(A_{m}\right)^{\mathrm{p}, \mathrm{RTD}}=\Gamma_{n-1}^{\mathrm{p}, \mathrm{RTD}}-\Gamma_{n}^{\mathrm{p}, \mathrm{RTD}}$ are obtained through

$$
\begin{aligned}
& \Gamma_{n}^{\mathrm{p}, \mathrm{RTD}}=\operatorname{Pr}\left(\log \left(1+\sum_{i=1}^{n} \frac{T_{i}^{\mathrm{p}} G_{i}^{\mathrm{pp}}}{1+T_{i}^{\mathrm{s}} G_{i}^{\mathrm{sp}}}\right)<R^{\mathrm{p}}\right) \\
& \simeq \operatorname{Pr}\left(\log \left(1+\sum_{i=1}^{n} \Omega_{i}^{\mathrm{p}}\right) \leq R^{\mathrm{p}}\right)=\operatorname{Pr}\left(\sum_{i=1}^{n} \Omega_{i}^{\mathrm{p}} \leq e^{R^{\mathrm{p}}}-1\right) \\
& =\int_{0}^{e^{R^{\mathrm{p}}}-1} \int_{0}^{e^{R^{\mathrm{p}}}-1} \ldots \int_{0}^{e^{R^{\mathrm{p}}}-1} f_{\Omega_{1}^{\mathrm{p}}\left(x_{1}\right) \ldots f_{\Omega_{n-1}^{\mathrm{p}}}\left(x_{n-1}\right) \times}^{n-1} \operatorname{Pr}\left(\Omega_{n}^{\mathrm{p}} \leq y-\sum_{i=1}^{x_{i}} x_{i}\right) \mathrm{d} x_{1} \mathrm{~d} x_{2} \ldots \mathrm{d} x_{n-1} \\
& =\left(\prod_{i=1}^{n-1} \beta_{i}\right) \int_{0}^{e^{R^{\mathrm{p}}}-1} \int_{0}^{e^{R^{\mathrm{p}}}-1} e^{-\left(\sum_{i=1}^{n-1} \beta_{i} x_{i}\right)} \times \\
& =\prod_{i=1}^{n-1}\left(1-e^{-\beta_{i}\left(e^{R^{\mathrm{p}}}-1\right)}\right) \\
& -e^{-\beta_{n}\left(e^{R^{\mathrm{p}}}-1\right)} \frac{\prod_{i=1}^{n-1} \beta_{i}}{\prod_{i=1}^{n-1}\left(\beta_{i}-\beta_{n}\right)}\left(\prod_{i=1}^{n-1}\left(1-e^{-\left(\beta_{i}-\beta_{n}\right)\left(e^{R^{\mathrm{p}}}-1\right)}\right)\right) .
\end{aligned}
$$

Here, the inequality follows from (9) and the equalities come from multi-dimensional integration and some manipulations. Using (10), we can find $\Gamma_{n}^{\mathrm{p}, \mathrm{RTD}}, \operatorname{Pr}(\text { Outage })^{\mathrm{p}, \mathrm{RTD}}$ and $\operatorname{Pr}\left(A_{m}\right)^{\mathrm{p}, \mathrm{RTD}}$ and, consequently, the outage-limited throughput, i.e., (8).

\section{B. INR HARQ protocol}

As mentioned before, the INR protocol is based on an aggressive codeword transmission in the first round, sending further parity bits in the retransmissions and combining all received representations of the signal at the receiver. Therefore, the results of, e.g., [18], [20] can be used to find the PU achievable rate random variable as

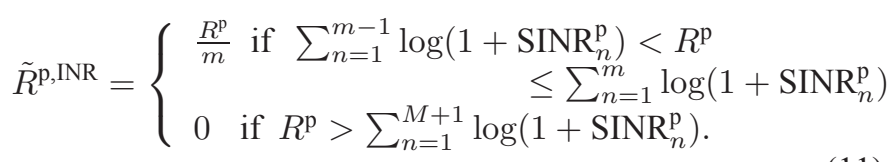

In this way, the only modifications that are required for studying the system performance in the presence of the INR HARQ is to replace the probabilities $\operatorname{Pr}\left(A_{m}\right)^{\mathrm{p}, \mathrm{RTD}}, \operatorname{Pr}(\text { Outage })^{\mathrm{p}, \mathrm{RTD}}$ and $\Gamma_{n}^{\mathrm{p}, \mathrm{RTD}}$ by

$$
\begin{gathered}
\operatorname{Pr}\left(A_{m}\right)^{\mathrm{p}, \mathrm{INR}}=\operatorname{Pr}\left(\sum_{i=1}^{m-1} \log \left(1+\frac{T_{i}^{\mathrm{p}} G_{i}^{\mathrm{pp}}}{1+T_{i}^{\mathrm{s}} G_{i}^{\mathrm{sp}}}\right)<R^{\mathrm{p}} \leq\right. \\
\left.\sum_{i=1}^{m} \log \left(1+\frac{T_{i}^{\mathrm{p}} G_{i}^{\mathrm{pp}}}{1+T_{i}^{\mathrm{s}} G_{i}^{\mathrm{sp}}}\right)\right), \\
\operatorname{Pr}(\text { Outage })^{\mathrm{p}, \mathrm{INR}}=\operatorname{Pr}\left(\sum_{i=1}^{M+1} \log \left(1+\frac{T_{i}^{\mathrm{p}} G_{i}^{\mathrm{pp}}}{1+T_{i}^{\mathrm{s}} G_{i}^{\mathrm{sp}}}\right)<R^{\mathrm{p}}\right),
\end{gathered}
$$

and

$$
\Gamma_{n}^{\mathrm{p}, \mathrm{INR}}=\operatorname{Pr}\left(\sum_{i=1}^{n} \log \left(1+\frac{T_{i}^{\mathrm{p}} G_{i}^{\mathrm{pp}}}{1+T_{i}^{\mathrm{s}} G_{i}^{\mathrm{sp}}}\right)<R^{\mathrm{p}}\right) .
$$

The rest of the arguments, such as Theorem 1 and Lemma 1, remain the same. Particularly, we can use (6), (14) and $\log (1+x) \rightarrow x$ for small $x$ 's, to show that the performance of the INR protocol converges to the one in the RTD at low SNRs. Therefore, the low-SNR approximation of Lemma 1 is valid for the INR as well. In the following, we study the system performance for the RTD and INR HARQ protocols.

\section{Simulation RESUlts}

The simulation results are obtained for the fixed PU (re)transmission powers and $R^{\mathrm{p}}=0.5$, unless otherwise stated. The throughput is presented in nats-per-channel-use (npcu). Moreover, the Rayleigh-fading channel parameters, e.g., $\lambda^{\mathrm{pp}}$, are set to 1 . The simulation results are presented as follows.

Considering the RTD HARQ protocol, Fig. 2 demonstrates the SU throughput versus the PU outage probability constraint. The results are obtained for $T^{\mathrm{p}}=T^{\text {peak }}=3.2$ and 10 which, as the noise variances are set to 1 , correspond to transmission SNRs 5 and $10 \mathrm{~dB}$, respectively ${ }^{1}$. The figure indicates that, depending on the PU outage probability constraint and its data transmission rate/power, there might be cases where no spectrum sharing is permitted. However, as the PU outage probability constraint gets relaxed the spectrum sharing becomes possible leading to positive SU throughput. Moreover, the PU robustness to the SU activity increases with the number of retransmissions. Therefore, increasing the number of retransmissions results in higher SU throughput. Moreover, although not included, the same trend is observed

\footnotetext{
${ }^{1}$ The reason we do not present the powers in $\mathrm{dB}$ is that in some cases the optimal power terms are found to be zero which can not be presented in log-scale.
} 


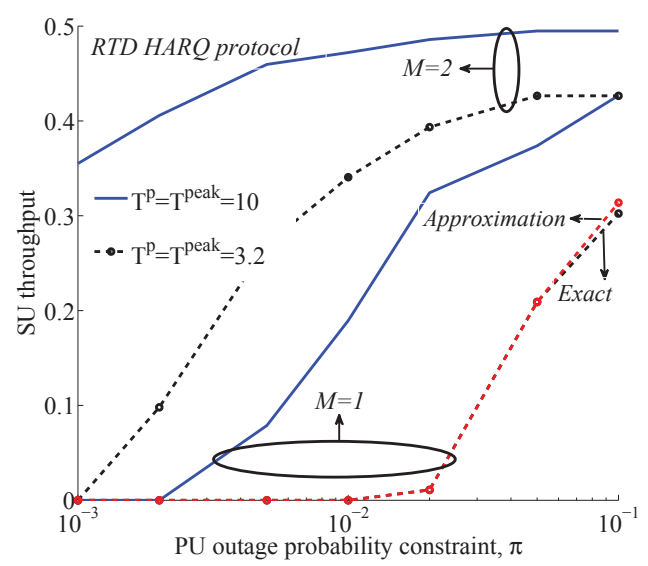

Figure 2. The secondary user throughput vs the primary user outage probability constraint, RTD protocol, $R^{\mathrm{p}}=0.5$. The red dashed curve shows the throughput achieved via the approximation scheme of Lemma 1 with $T^{\mathrm{p}}=T^{\text {peak }}=3.2, M=1$.

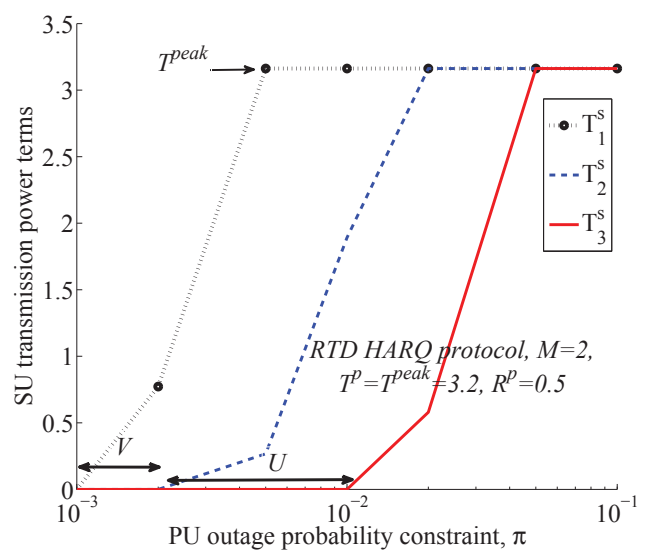

Figure 3. The SU optimal power terms vs the primary user outage probability constraint, RTD protocol, a maximum of $M=2$ retransmissions, $T^{\mathrm{p}}=$ $T^{\text {peak }}=3.2, R^{\mathrm{p}}=0.5$.

in the cases with a higher number of retransmissions. Finally, the figure shows an example for the accuracy of approximation approach of Lemma 1 . As it can be seen, there is very high accuracy for the approximation scheme of Lemma 1 at low SNRs. However, it should be mentioned that the rest of the simulation results are obtained by numerical calculation of the probabilities with no approximation.

The validity of Theorem 1 is investigated in Figs. 3-4 where the SU optimal transmission powers are obtained for different HARQ protocols. In harmony with Theorem 1, the SU sends the data with higher powers in the first PU (re)transmission rounds. Moreover, depending on the channel condition, it may occur that the SU turns off in the last retransmission (region $\mathrm{U}$ in Fig. 3) or in the two last retransmission rounds (region $\mathrm{V}$ in Fig. 3). Also, as the $\mathrm{SU}$ peak power constraint gets relaxed, i.e., $T^{\text {peak }}$ increases, more powers are given to the first retransmission rounds and meanwhile the SU power terms associated with the last PU retransmissions decreases, so that the PU outage probability constraint is satisfied (Fig. 4).

Figure 5 studies the SU throughput for the INR protocol and compares the results with the ones in the RTD. Compared to the RTD scheme, higher throughput is achieved by the SU

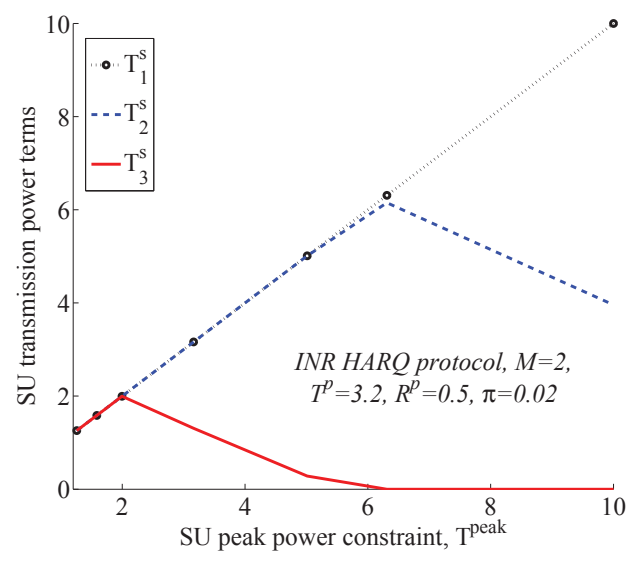

Figure 4. The SU optimal power terms vs the SU peak power constraint, INR protocol, a maximum of $M=2$ retransmissions, $T^{\mathrm{p}}=3.2, R^{\mathrm{p}}=0.5$, $\pi=0.02$.

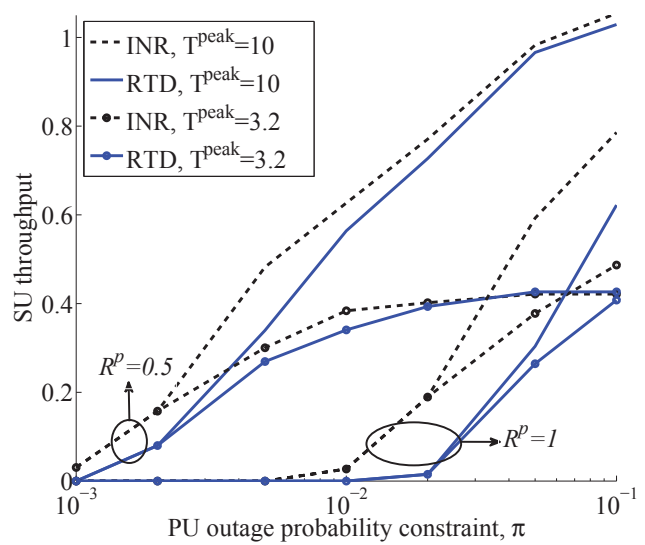

Figure 5. The secondary user throughput for different HARQ protocols, $T^{\mathrm{p}}=$ $10, M=2$.

when the INR HARQ is utilized by the PU. Also, with hard outage probability constraints, i.e., at low values of $\pi$, the PU outage probability is the main constraint determining the SU throughput. However, as the PU outage probability constraint gets relaxed, the SU peak transmission power constraint becomes more important affecting the SU throughput. Therefore, the SU throughput becomes less dependent of the PU status and, as seen in the figure, the throughput of the RTD- and INR-based schemes converges at high values of $\pi$.

On the effect of spatial correlations: Figures 2-5 are obtained based on the assumption that the fading channels are spatially independent. However, depending on the environmental properties, realistic channels may not be independent [22], [23]. Therefore, it is important to study the effect of spatial correlations on the system performance. For this reason, we consider the spatially-correlated Rayleigh-fading model where the relations between every two fading random variables $H^{\mathrm{ij}}$ and $H^{\mathrm{kl}},(k, l) \neq(i, j)$, are modeled by

$$
H^{\mathrm{ij}}=\delta^{\mathrm{ijkl}} H^{\mathrm{kl}}+\sqrt{1-\delta^{\mathrm{ijkl}}{ }^{2}} \epsilon, \epsilon \sim \mathcal{C N}(0,1) .
$$

Here, $\delta^{\mathrm{ijkl}}$ is a known correlation factor modeling the two variable dependencies. This is a well-established model considered in the literature for different applications [23], [24]. In this 


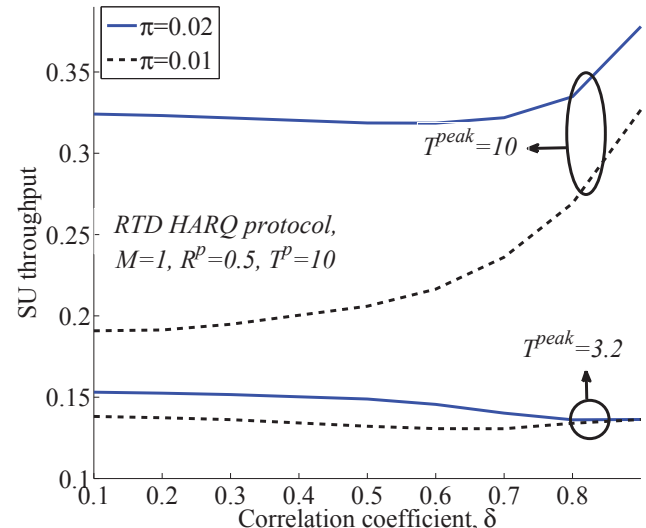

Figure 6. The secondary user throughput for different spatial correlation conditions, RTD HARQ, $M=1, T^{\mathrm{p}}=10, R^{\mathrm{p}}=0.5$.

way, the joint pdf of the gains is found as

$$
f_{G^{\mathrm{ij}}, G^{\mathrm{kl}}}(x, y)=\frac{e^{-\frac{x+y}{1-\delta_{\mathrm{j} \mathrm{kl} l^{2}}}}}{1-\delta_{\mathrm{ijk} l^{2}}} I_{0}\left(\frac{2 \delta^{\mathrm{ijkl}} \sqrt{x y}}{1-\delta^{\mathrm{ijkl}}{ }^{2}}\right),
$$

where $I_{0}($.$) is the zeroth-order modified Bessel function of$ the first kind [23], [25]. Here, the interesting point is that, according to the above discussions, the only parameters that are affected by the spatially-correlated fading model are the probability terms which should be recalculated based on (16). However, the rest of the arguments are independent of the fading model.

Setting $\delta^{\text {ssps }}=\delta^{\text {ppsp }}=\delta, \delta^{\text {ppss }}=0$, Fig. 6 investigates the effect of spatial dependencies on the system performance. The results indicate that in the practical range of correlation conditions the fading dependencies do not affect the system performance, in the sense that the SU throughput changes are negligible at low $\delta$ 's. Therefore, the spatially-independency is an acceptable assumption for the analytical performance analysis. On the other hand, the data transmission efficiency of the spectrum sharing networks is considerably affected by high correlation coefficients, the range which is not of practical interest. Finally, depending on the parameter settings, the SU throughput may increase or decrease at $\delta \sim 1$.

\section{CONCLUSION}

This paper studied the outage-limited throughput of the spectrum sharing networks using HARQ protocols and adaptive power allocation. The results were obtained for both independent and spatially-correlated fading channels. The numerical and analytical results show that, using HARQ and adaptive power allocation, the maximum outage-limited throughput is achieved by combination of simultaneous transmission and interference-avoiding spectrum sharing paradigms. This is an interesting result providing an appropriate connection between the papers considering one of these paradigms. Also, we developed tight approximations for the performance analysis of HARQ-based spectrum sharing setups. The performance of the spectrum sharing networks is shown to be not sensitive to spatial correlation, within the practical range of interest. Finally, implementation of HARQ protocols and adaptive power allocation leads to considerable performance improvement in spectrum sharing networks.

\section{REFERENCES}

[1] S. A. R. Zaidi, D. C. McLernon, and M. Ghogho, "Outage probability analysis of cognitive radio networks under self-coexistence constraint," in CISS, 2010, pp. 1-6.

[2] S. Akin and M. C. Gursoy, "Cognitive radio transmission under QoS constraints and interference limitations," EURASIP J. on Wireless Commun. and Net., vol. 2012:301, 2012.

[3] S. M. Mishra, et. al, "Cooperative sensing among cognitive radios," in ICC, vol. 4, June 2006, pp. 1658-1663.

[4] S. A. Jafar and S. Srinivasa, "Capacity limits of cognitive radio with distributed and dynamic spectral activity," IEEE J. on Sel. Areas in Commun., vol. 25, no. 3, pp. 529-537, April 2007.

[5] N. Devroye, et. al, "Cognitive radio networks," IEEE Signal Processing Magazine, vol. 25, no. 6, pp. 12-23, Nov. 2008.

[6] M. Gastpar, "On capacity under receive and spatial spectrum-sharing constraints," IEEE Trans. on Info. Theory, vol. 53, no. 2, pp. 471-487, Feb. 2007.

[7] Y. Xing, et. al, "Dynamic spectrum access with QoS and interference temperature constraints," IEEE Trans. on Mobile Computing, vol. 6, no. 4, pp. 423-433, April 2007.

[8] X. Kang, et. al, "Optimal power allocation for fading channels in cognitive radio networks: Ergodic capacity and outage capacity," IEEE Trans. on Wireless Commun., vol. 8, no. 2, pp. 940-950, Feb. 2009.

[9] A. Ghasemi and E. S. Sousa, "Fundamental limits of spectrum-sharing in fading environments," IEEE Trans. on Wireless Commun., vol. 6, no. 2, pp. 649-658, Feb. 2007.

[10] C.-X. Wang, et. al, "On capacity of cognitive radio networks with average interference power constraints," IEEE Trans. on Wireless Commun., vol. 8, no. 4, pp. 1620-1625, April 2009.

[11] L. Musavian and S. Aissa, "Capacity and power allocation for spectrumsharing communications in fading channels," IEEE Trans. on Wireless Commun., vol. 8, no. 1, pp. 148-156, Jan. 2009.

[12] W. C. Ao and K. C. Chen, "End-to-end HARQ in cognitive radio networks," in $W C N C, 2010$, pp. 1-6.

[13] R. Narasimhan, "Hybrid-ARQ interference channels with receiver cooperation," ICC, pp. 1-5, May 2010.

[14] R. A. Tannious and A. Nosratinia, "Cognitive radio protocols based on exploiting hybrid ARQ retransmissions," IEEE Trans. on Wireless Commun., vol. 9, no. 9, pp. 2833-2841, 2010.

[15] K. Eswaran, et. al, "Bits through ARQs: Spectrum sharing with a primary packet system," in ISIT, June 2007, pp. 2171-2175.

[16] B. Makki and T. Eriksson, "On the average rate of HARQ-based quasistatic spectrum sharing networks," IEEE Trans. on Wireless Commun., vol. 11, no. 1, pp. 65-77, Jan. 2012.

[17] B. Makki, A. Graell i Amat, and T. Eriksson, "HARQ feedback in spectrum sharing networks," IEEE Commun. Lett., vol. 16, no. 9, pp. 1337-1340, Sept. 2012.

[18] B. Makki and T. Eriksson, "On the average rate of quasi-static fading channels with ARQ and CSI feedback," IEEE Commun. Letters, vol. 14, no. 9, pp. 806-808, June 2010.

[19] B. Makki, A. Graell i Amat, and T. Eriksson, "Power allocation in repetition time diversity hybrid automatic repeat request feedback," in WCNC, 2012, pp. 2329-2334.

[20] D. Tuninetti, "On the benefits of partial channel state information for repetition protocols in block fading channels," IEEE Trans. Inf. Theory, vol. 57, no. 8, pp. 5036-5053, Aug. 2011.

[21] B. Makki, A. Graell i Amat, and T. Eriksson, "Green communication via power-optimized HARQ protocols," IEEE Trans. on Veh. Tech., 2013, in press.

[22] T. Eriksson and T. Ottosson, "Compression of feedback for adaptive transmission and scheduling," Proceedings of the IEEE, vol. 95, no. 12, pp. 2314-2321, Dec. 2007.

[23] H. A. Suraweera, P. J. Smith, and M. Shafi, "Capacity limits and performance analysis of cognitive radio with imperfect channel knowledge," IEEE Trans. Veh. Technol., vol. 59, no. 4, pp. 1811-1822, 2010.

[24] K. Huang, R. W. Heath, and J. G. Andrews, "Limited feedback beamforming over temporally-correlated channels," IEEE Trans. Signal Process., vol. 57, no. 5, pp. 1959-1975, 2009.

[25] C. Tellambura and A. D. S. Jayalath, "Generation of bivariate rayleigh and nakagami-m fading envelopes," IEEE Commun. Lett., vol. 4, no. 5, pp. 170-172, 2000 . 